\title{
Homocystinuria without methylmalonic aciduria
}

INSERM

\section{Source}

INSERM. (1999). Orphanet: an online rare disease and orphan drug data base.

Homocystinuria without methylmalonic aciduria. ORPHA:622

Homocystinuria without methylmalonic aciduria is an inborn error of vitamin B12

(cobalamin) metabolism characterized by megaloblastic anemia, encephalopathy and,

sometimes, developmental delay, and associated with homocystinuria and

hyperhomocysteinemia. There are three types of homocystinuria without methylmalonic aciduria; cblE, cblG and cblD-variant 1 (cblDv1). 